ARTICLE

\title{
Flexible transition metal dichalcogenide nanosheets for band-selective photodetection
}

\author{
Dhinesh Babu Velusamy', Richard Hahnkee Kim¹, Soonyoung Cha², June Huh ${ }^{3}$, Reza Khazaeinezhad ${ }^{4}$, \\ Sahar Hosseinzadeh Kassani ${ }^{4}$, Giyoung Song ${ }^{1}$, Suk Man Cho', Sung Hwan Cho', Ihn Hwang ${ }^{1}$, Jinseong Lee \\ Kyunghwan $\mathrm{Oh}^{4}$, Hyunyoug $\mathrm{Choi}^{2} \&$ Cheolmin Park ${ }^{1}$
}

The photocurrent conversions of transition metal dichalcogenide nanosheets are unprecedentedly impressive, making them great candidates for visible range photodetectors. Here we demonstrate a method for fabricating micron-thick, flexible films consisting of a variety of highly separated transition metal dichalcogenide nanosheets for excellent band-selective photodetection. Our method is based on the non-destructive modification of transition metal dichalcogenide sheets with amine-terminated polymers. The universal interaction between amine and transition metal resulted in scalable, stable and high concentration dispersions of a single to a few layers of numerous transition metal dichalcogenides. Our $\mathrm{MoSe}_{2}$ and $\mathrm{MoS}_{2}$ composites are highly photoconductive even at bending radii as low as $200 \mu \mathrm{m}$ on illumination of near infrared and visible light, respectively. More interestingly, simple solution mixing of $\mathrm{MoSe}_{2}$ and $\mathrm{MoS}_{2}$ gives rise to blended composite films in which the photodetection properties were controllable. The $\mathrm{MoS}_{2} / \mathrm{MoSe}_{2}$ (5:5) film showed broad range photodetection suitable for both visible and near infrared spectra.

\footnotetext{
${ }^{1}$ Department of Materials Science and Engineering, Yonsei University, Seoul 120-749, Korea. ${ }^{2}$ School of Electrical and Electronic Engineering, Yonsei University, Seoul 120-749, Korea. ${ }^{3}$ Department of Chemical and Biological Engineering, Korea University, Anam-dong, Seongbuk-gu, Seoul 136-713, Korea. ${ }^{4}$ Photonic Device Physics Laboratory, Institute of Physics and Applied Physics, Yonsei University, Seoul 120-749, Korea. Correspondence and requests for materials should be addressed to C.P. (email: cmpark@yonsei.ac.kr) or H.C. (email: hychoi@yonsei.ac.kr).
} 
$\mathrm{T}$ wo-dimensional (2D) nanosheets of transition metal dichalcogenides (TMDs) such as $\mathrm{MoS}_{2}, \mathrm{WS}_{2}$ and $\mathrm{MoSe}_{2}$ have been of great attraction due to their intriguing photoelectronic properties associated with the 2D confined chemical structures from insulators, direct bandgap semiconductors to metals $s^{1-3}$. These materials have potential for applications in electronics, optics, energy conversion and storage ${ }^{4-11}$. In particular, the photocurrent conversion at the characteristic photon energies corresponding to their material-dependent energy band gaps is impressive, making them great candidates for wavelength-selective photodetectors ${ }^{12-18}$. Excellent visible photodetection has been, for instance, achieved with devices containing single or few layered $\mathrm{MoS}_{2}$, as well as $\mathrm{WS}_{2}$ fabricated by mechanical exfoliation with Scotch film ${ }^{19}$; convenient but hardly applicable for arrays of the devices.

These promising photoconversion performances of various $2 \mathrm{D}$ TMDs is driving a demand for their technological implementation. Furthermore, considering the mechanically flexible nature of the $2 \mathrm{D} \mathrm{TMDs}^{20,21}$, arrays of the photodetectors on either plastic or paper would be beneficial for wearable and patchable applications. Several challenges are, however, ahead for the successful realization. Most importantly, a scalable and universal process suitable for various TMDs irrespective of their detailed chemical structures is required for thin uniform film fabrication on diverse substrates. The method should involve efficient exfoliation of the sheets of 2D TMDs from stacked bulk samples, as well as prevention of re-aggregation of the sheets on film formation. In addition, individual or a few layers of $2 \mathrm{D}$ TMDs should be well-connected with each other in the film to readily develop conducting pathways between two electrodes for efficient photocarrier transport.

Many studies were devoted to liquid phase exfoliation and stabilization of TMDs ${ }^{22-33}$ not only because the solvent medium offers an extra driving force for the separation of the sheets but also because 2D TMDs dispersed in solvent can be suitable for various solution-based film processes such as spin-coating, dip-coating and layer-by-layer assembly. To further promote the separation of the sheets, additional interactions with TMDs were required, including ion intercalation ${ }^{22}$, surfactant-driven interaction $^{29}$ and highly boiled medium ${ }^{33}$. Some of the previous approaches are promising and scalable, but limited to specific solvent medium. In addition, photoelectronic properties of mechanically flexible TMD films have not been extensively investigated, in particular, comparable with those with mechanically exfoliated sheets. The non-destructive dispersion strategy with synthetic polymers can be additionally beneficial due to their long and flexible chains that adhere to the surface of TMDs and thus provide sufficient physical gaps between two sheets to mitigate the strong van der Waal interactions of the sheets $^{34}$. We envisioned that end-functionalized polymers can meet the requirements aforementioned with end-functional groups universally interactive with various TMDs. Flexible polymer chains dangled with end-functional moieties firmly anchored on the surface of TMDs in solvents can allow for good dispersion of the sheets with their minimum amount, leading to flexible composite films with many conducting pathways.

Here we present mechanically flexible TMD nanosheets for band-selective photodetection. Our strategy of modifying TMDs with amine-terminated polymers successfully offers a scalable platform suitable for fabricating various flexible TMD/polymer composite films in which few-layer TMD nanosheets are properly separated from each other and embedded in a polymer matrix. The pivotal process for the platform is the extremely efficient liquid exfoliation of TMDs with primary amine-terminated polymers whose end-functional amines are firmly anchored on the surface of TMDs due to strong Lewis acid-base interaction between transition metal and non-pair electrons of amine, allowing us to develop various uniform composite films with numerous combinations of TMD nanosheets and endfunctionalized polymers. Films of $\mathrm{MoSe}_{2}$ nanosheets with amine-terminated poly(styrene) $\left(\mathrm{PS}-\mathrm{NH}_{2}\right)$ exhibited excellent photodetection with an ON/OFF photocurrent ratio of $10^{5}$, a detectivity of $4 \times 10^{12}$ Jones, a responsivity of $16 \mathrm{~A} \mathrm{~W}^{-1}$ and a response time of $100 \mathrm{~ms}$ on 1,064-nm illumination, even when the films were severely deformed at a bending radius of $\sim 200 \mu \mathrm{m}$. Moreover, simple solution mixing of $\mathrm{MoSe}_{2}$ and $\mathrm{MoS}_{2}$ modified with $\mathrm{NH}_{2}$-PS offers an extremely convenient route for tuning the photodetection properties, and thus a blended composite film allows for broad range photodetection from visible to near infrared (NIR). The band-selective photodetection of our blended films is also evidenced by thorough investigation of photo-induced, carrier-relaxation dynamics.

\section{Results}

Universal exfoliation of TMDs with amine-terminated polymers. In this work, we achieved successful exfoliation of numerous TMD nanosheets such as $\mathrm{MoS}_{2}, \mathrm{WS}_{2}, \mathrm{MoSe}_{2}, \mathrm{WSe}_{2}, \mathrm{ReS}_{2}, \mathrm{ZrTe}_{2}$ and $\mathrm{NbSe}_{2}$ with amine-terminated polymers. The principle is based on the Lewis-like acid-base interaction ${ }^{22,35}$ between the transition metal and primary amine, as shown in the scheme in Fig. 1. The representative amine-terminated polymers employed here are glassy PS, poly(methyl methacrylate), rubbery poly(butadiene), semi-crystalline poly(ethylene), poly(ethylene oxide) (PEO) and poly(styrene- $b$-isoprene) copolymer. The interaction between the donated lone pairs of nitrogen atoms and electron-accepting metal atoms was evidenced by Fourier transform infrared spectroscopic (FT-IR) for the case of $\mathrm{MoSe}_{2}$ modified with PS- $\mathrm{NH}_{2}$. (Supplementary Note 1) The characteristic peak at $1,600 \mathrm{~cm}^{-1}$ corresponding to neat $\mathrm{NH}_{2}$ was significantly shifted to $\sim 1,650 \mathrm{~cm}^{-1}$, which resulted from the $\mathrm{N}-\mathrm{H}$ bonds bent due to the transition metal-amine interaction, as shown in Fig. 1. Upshift of $\mathrm{N}-\mathrm{H}$ mode occurred when primary amines interact with transition metals ${ }^{36,37}$. In addition, the computation of IR spectra for model systems (PS- $\mathrm{NH}_{2}$, $\mathrm{MoSe}_{2} / \mathrm{PS}-\mathrm{NH}_{2}$ ) based on density functional theory frequency calculation also shows that the peak corresponding to bending vibration of the $\mathrm{NH}_{2}$ in the absence of $\mathrm{MoSe}_{2}$ was shifted to higher frequency in the $\mathrm{MoSe}_{2} / \mathrm{PS}-\mathrm{NH}_{2}$ bound state, which consolidates our experimental observation (Supplementary Fig. 1).

Amine groups of polymers efficiently interact with transition metals when surface of TMD nanosheets is exposed upon sonication step. Simultaneously, flexible polymer chains from the anchored amine on the TMD surface are fully extended in their good solvent medium. Firmly anchored polymer chains on the TMD surface prevent the exfoliated nanosheets from reaggregating on solvent evaporation. It should be noted that our process does not involve the intercalation of TMD nanosheets, different from ones previously developed with metal ions ${ }^{22-25}$ (Supplementary Fig. 2; Supplementary Note 1). The successful interaction between amine groups of polymers and transition metals in the TMD nanosheets is confirmed by density functional tight binding ${ }^{38-40}$ and Born-Oppenheimer molecular dynamics simulation (Supplementary Fig. 3). Combined with conventional sonication and centrifugation processes, we were able to exfoliate the bulk TMDs into few-layer nanosheets on a large scale, as shown in the photograph in Fig. 1.

To demonstrate the effectiveness of our strategy, we extensively investigated the dispersion of $\mathrm{MoSe}_{2}$ nanosheets with PS- $\mathrm{NH}_{2}$ (Supplementary Note 2). The suspension of bulk $\mathrm{MoSe}_{2}$ with $1 \mathrm{mg} \mathrm{ml}^{-1}$ PS- $\mathrm{NH}_{2}$ in Fig. 2a exhibits the characteristic absorbance spectrum of $\mathrm{MoSe}_{2}$ (blue colour) after optimization 


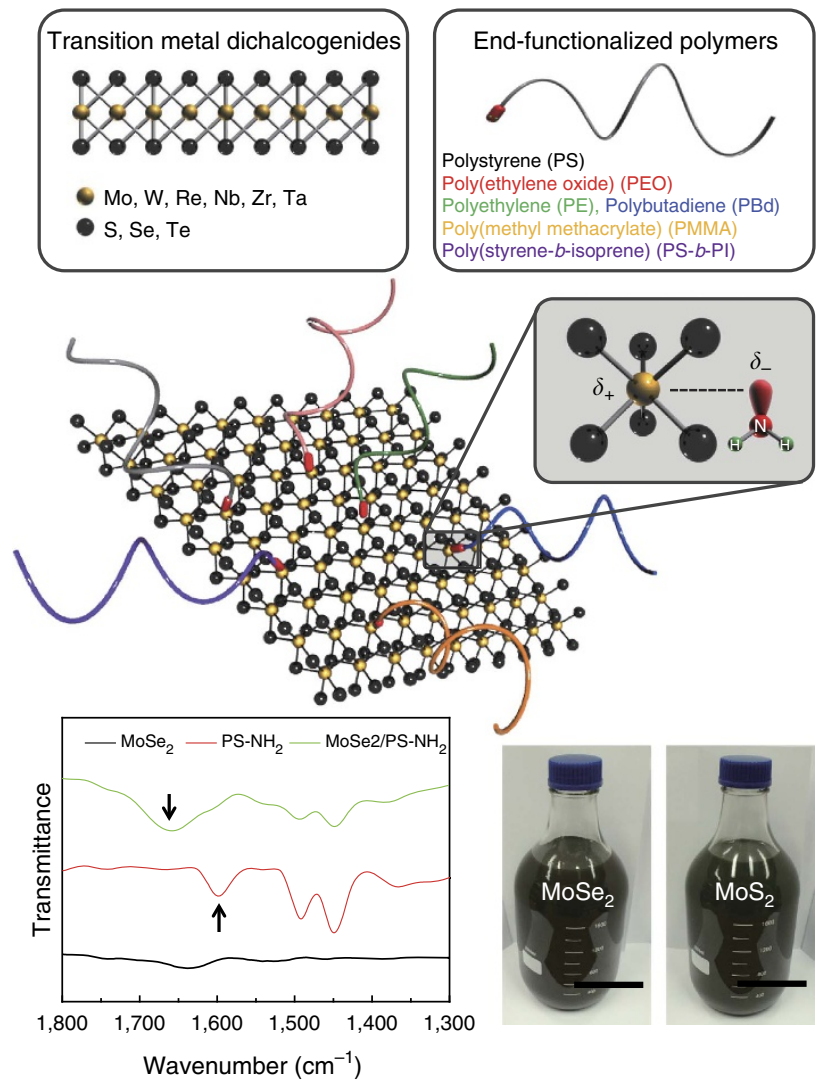

Figure 1 | Liquid phase exfoliation of TMDs with end-functionalized polymers. Schematic representation of the proposed mechanism for the exfoliation of various TMDs using different types of amine-terminated end-functionalized polymers in organic solvents. Hexagonal layers of transition metal atoms $(\mathrm{M})$ sandwiched between two layers of chalcogenides $(X)$ are represented by a stoichiometry of $M X_{2}$ by the yellow and grey spheres, respectively. The amine-terminated polymers are simplified for clarity. Interaction between the lone electron pairs of nitrogen atoms and the electron-accepting metal atoms weakens the self layer-layer attraction and the polymer chains provide further separation between the layers, thereby ensuring good exfoliation and dispersion. FT-IR spectra of $\mathrm{MoSe}_{2}, \mathrm{PS}-\mathrm{NH}_{2}$ and $\mathrm{MoSe}_{2}-\mathrm{PS}-\mathrm{NH}_{2}$ are shown. Few-layer $\mathrm{MoSe}_{2}$ and $\mathrm{MoS}_{2}$ nanosheets dispersed on the $2-I$ scale are apparent in the photographs (Scale bar, $5 \mathrm{~cm}$ ).

of the sonication time, initial $\mathrm{MoSe}_{2}$ concentration and centrifugation rate (Supplementary Fig. 4). Different from pure PS- $\mathrm{NH}_{2}$, discernible peaks were observed at 800 and $690 \mathrm{~nm}$, which are similar to the characteristic peaks of exfoliated $\mathrm{MoSe}_{2}$ (red colour) obtained by other methods without PS- $\mathrm{NH}_{2}$, confirming the stable exfoliation and dispersion of $\mathrm{MoSe}_{2}$ in toluene ${ }^{26,29,41}$. The increased absorbance at the short wavelength side is attributed to the background scattering arising from TMD nanosheets with $2 \mathrm{D}$ anisotropic shape ${ }^{26,29}$. To further evidence that the strong absorption peaks in Fig. 2a are attributed to the A or B exciton peaks of $\mathrm{MoSe}_{2}$, we performed the absorbance experiments at wavelength-selective system (Supplementary Fig. 5) and the results are the same as Fig. 2a (Supplementary Fig. 6). Additional experiments using X-ray photoelectron spectroscopy, Raman spectroscopy and X-ray diffraction further confirmed the exfoliation and dispersion of $\mathrm{MoSe}_{2}$ nanosheets (Supplementary Fig. 7). The characteristic direct bandgap structure of mono and a few layered $\mathrm{MoSe}_{2}$ nanosheets was evidenced by photoluminescence (PL) measurement (Supplementary Fig. 8). The PL peak centred at $\sim 808 \mathrm{~nm}$
$(1.54 \pm 0.01 \mathrm{eV})$ from A excitons of $\mathrm{MoSe}_{2}$ is attributed to an indirect-to-direct bandgap transition, which occurs at the $\mathrm{K}$ high symmetry point of the Brillouin zone associated with the quantum confinement in the perpendicular direction ${ }^{16,17,42-44}$. The PL emission peak from $\mathrm{MoSe}_{2}$ modified with PS- $\mathrm{NH}_{2}$ is consistent with that from either mechanically exfoliated or CVD synthesized monolayer and the results suggest that the observed PL arises from the intrinsic electronic properties of monolayer $\mathrm{MoSe}_{2}$ and our exfoliated $\mathrm{MoSe}_{2}$ nanosheets possess semiconducting $2 \mathrm{H}$ phase with hexagonal prismatic $\mathrm{D}_{3 \mathrm{~h}}$ symmetry. The results we obtained with $\mathrm{MoSe}_{2}$ nanosheets modified with PS- $\mathrm{NH}_{2}$ were similarly observed with the nanocomposites of $\mathrm{MoS}_{2}$ with PS-NH , which confirms the universality of our method (Supplementary Fig. 9).

The effect of the molecular weight of PS- $\mathrm{NH}_{2}(9.5,25$ and $40 \mathrm{k}$, and $108 \mathrm{~kg} \mathrm{~mol}^{-1}$ ) on the exfoliation of $\mathrm{MoSe}_{2}$ additionally supports our arguments. For initial polymer concentrations of 1 and $5 \mathrm{mg} \mathrm{ml}^{-1}$, the absorbance nearly linearly decreased with increasing molecular weight of polymers due to the decrease of the total number of end-amine groups with increasing molecular weight of the polymers, as shown in Fig. 2b. Based on the results, $9.5 \mathrm{k}$ PS- $\mathrm{NH}_{2}$ was further utilized for the exfoliation and dispersions of the TMDs (Supplementary Fig. 10).

A key advantage of our method is the capability of dispersing $\mathrm{MoSe}_{2}$ in many solvents. Eight different solvents were examined, which were all good solvents for PS. The results were well-fitted on a single absorbance versus concentration plot of dispersed $\mathrm{MoSe}_{2}$, which implies that the $\mathrm{MoSe}_{2}$ nanosheets were uniformly dispersed without aggregation, as shown in Fig. 2c (Supplementary Figs 11 and 12). The maximum amount of dispersed $\mathrm{MoSe}_{2}$ as a function of the PS- $\mathrm{NH}_{2}$ concentration depends on the solvent surface energy and the degree of solubility of PS with solvents ${ }^{26,29,30,34}$ where the results are shown in Fig. 2d. The concentration of dispersed $\mathrm{MoSe}_{2}$ increases with increasing PS- $\mathrm{NH}_{2}$ concentration but rarely changes when the polymer concentration is $5 \mathrm{mg} \mathrm{ml}^{-1}$, indicating the saturation of interaction sites available on the TMD surface. Notably, for all of the solvents examined, at least $0.55 \mathrm{mg} \mathrm{ml}^{-1} \mathrm{MoSe}_{2}$ was stably dispersed and $\sim 1.6 \mathrm{mg} \mathrm{ml}^{-1} \mathrm{MoSe}_{2}$ was successfully exfoliated with our PS-NH $\mathrm{N}_{2}$ in NMP (Supplementary Fig. 13). The maximum yield percentage of the dispersed $\mathrm{MoSe}_{2}$ is $>12 \%$ in NMP. (Fig. 2d) Compared with previous works ${ }^{25-32}$, the maximum amount of dispersed $\mathrm{MoSe}_{2}$ is substantially high and, in particular, it should be noted that the scalable exfoliation of $\mathrm{MoSe}_{2}$ in various solvents was achieved without re-aggregation for a period of $>3$ weeks (Supplementary Fig. 14). As proposed in Fig. 1, other amine-terminated polymers also produced $\mathrm{MoSe}_{2}$ dispersed in various solvent medium, as shown in Fig. 2e. By choosing PEO, for example, a large amount of $\mathrm{MoSe}_{2}$ nanosheets was dispersed in polar media including ethanol and water. To confirm the universality of our method, we dispersed six additional TMDs including $\mathrm{MoS}_{2}, \mathrm{WS}_{2}, \mathrm{WSe}_{2}, \mathrm{ReS}_{2}, \mathrm{ZrTe}_{2}$ and $\mathrm{NbSe}_{2}$ with PS- $\mathrm{NH}_{2}$ in toluene where their absorbance spectra are shown in Fig. 2f (Supplementary Fig. 15) 29,41 .

The microstructures of the exfoliated nanosheets were investigated using both surface probe and electron microscopy, where the results show that the thickness of the single-layer $\mathrm{MoSe}_{2}$ was $1.0 \pm 0.15 \mathrm{~nm}$, which is thicker than that of pristine $\mathrm{MoSe}_{2}$ (ref. 16) $(\sim 0.7 \mathrm{~nm})$ due to the attached polymer chains on both sides of the nanosheet. The single crystalline nature ${ }^{45}$ of the single- and few-layer $\mathrm{MoSe}_{2}$ was preserved during the exfoliation process as well (Supplementary Fig. 16). Statistical analysis of both the lateral size and thickness of the exfoliated $\mathrm{MoSe}_{2}$ nanosheets (Supplementary Fig. 17) suggests that $\sim 70 \%$ of the $\mathrm{MoSe}_{2}$ nanosheets was one to three layers with broadly 

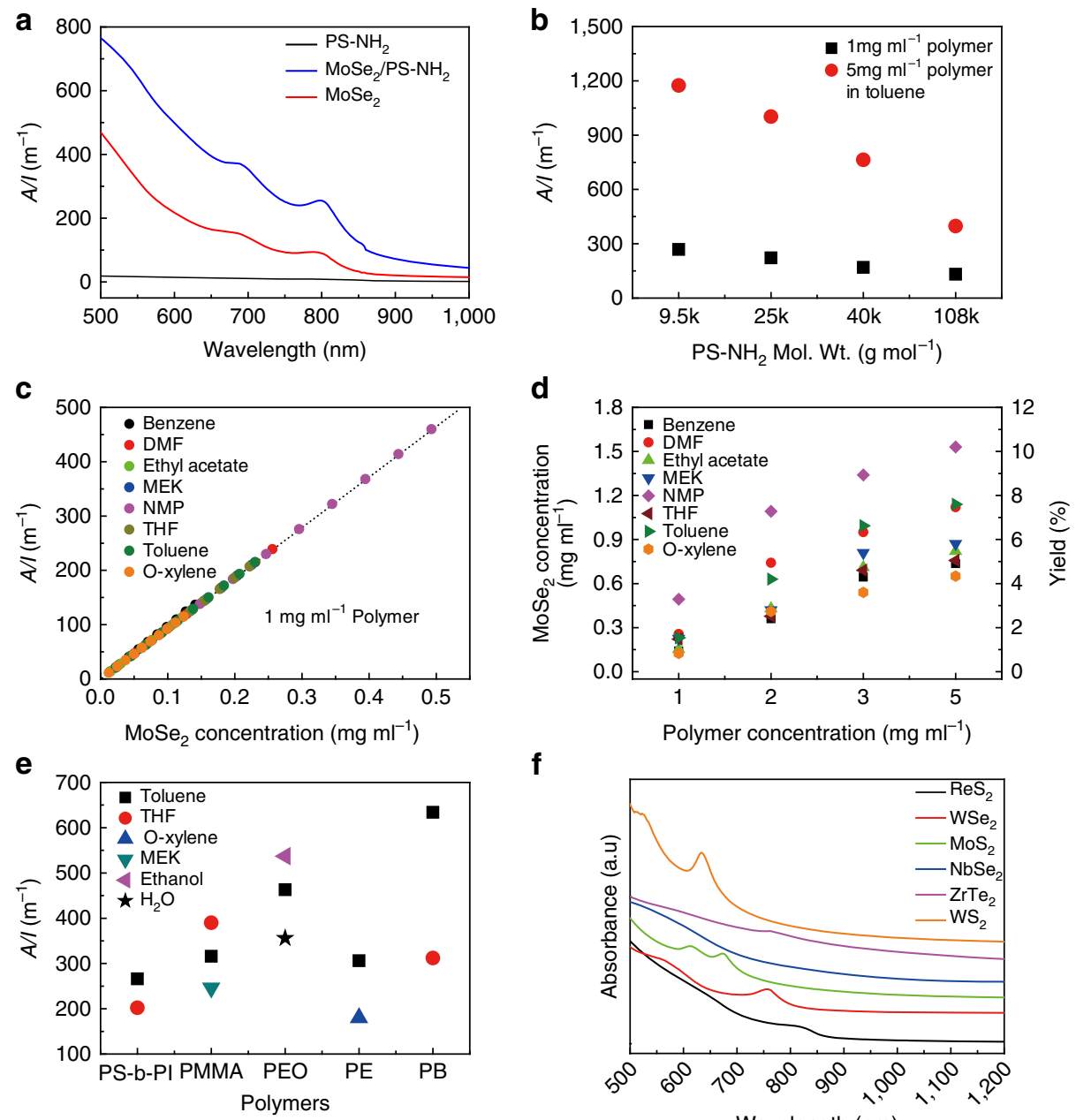

f

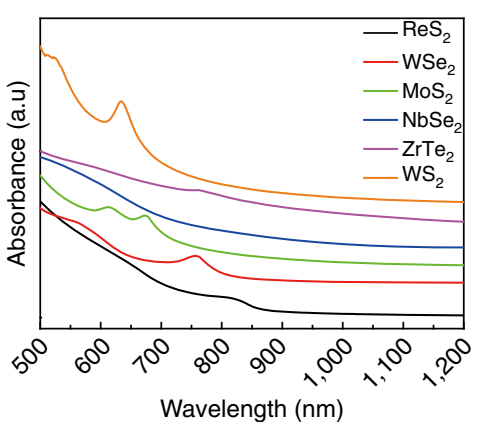

Figure 2 | Dispersion characteristics of TMD nanosheets in organic solvents. (a) Absorbance spectra of PS- $\mathrm{NH}_{2}$ (black), $\mathrm{MoSe}_{2}$ dispersed with PS-NH in toluene (blue) and $\mathrm{MoSe}_{2}$ dispersed without PS- $\mathrm{NH}_{2}$ in NMP (red). (b) Effect of the molecular weight of PS-NH $\mathrm{N}_{2}$ on the efficiency of the MoSe 2 dispersion in toluene characterized by the absorbance values of the dispersions at $800 \mathrm{~nm}$. The initial concentrations of $\mathrm{MoSe}_{2}$ and $\mathrm{PS}-\mathrm{NH}_{2}$ were kept constant at $10 \mathrm{mg} \mathrm{ml}^{-1}$ and $1 \mathrm{mg} \mathrm{ml}^{-1}$, respectively, for all of the molecular weights of PS- $\mathrm{NH}_{2}$ evaluated. Increasing the molecular weight of PS-NH $\mathrm{H}_{2}$ leads to a decrease of the efficiency of the $\mathrm{MoSe}_{2}$ dispersion. (c) Absorbance at a wavelength of $800 \mathrm{~nm}$ as a function of the MoSe $\mathrm{M}_{2}$ concentration in each solvent. The absorbance linearly increased with the amount of $\mathrm{MoSe}_{2}$ following Lambert-Beer behaviour, which implies uniform dispersion of MoSe $\mathrm{e}_{2}$ without aggregation in all of the solvents. (d) Plots of the concentration of $\mathrm{MoSe}_{2}$ as a function of the initial concentration of PS-NH $\mathrm{H}_{2}$ in different organic solvents. Yield of one to three layers (70\%) of the exfoliated $\mathrm{MoSe}_{2}$ nanosheets with $\mathrm{PS}-\mathrm{NH}_{2}$ as a function of the initial concentration of PS-NH $\mathrm{N}_{2}$ in different organic solvents are also shown. (e) Absorbance per cell length (proportional to the concentration of dispersed $\mathrm{MoSe}_{2}$ ) of $\mathrm{MoSe}_{2}$ dispersed in a range of solvents with a concentration of $3 \mathrm{mg} \mathrm{ml}^{-1}$ of the different amine-terminated polymers. Note that $\mathrm{PE}-\mathrm{NH}_{2}$ was dissolved in hot toluene and xylene. For $\mathrm{MoSe}_{2}$ dispersion in water with PEO- $\mathrm{NH}_{2}$, sonication was applied for $2 \mathrm{~h}$. (f) Absorbance spectra of the dispersions of different TMDs exfoliated in toluene with $\mathrm{PS}-\mathrm{NH}_{2}$. The absorbance spectra are vertically displaced for clarity.

distributed lateral dimensions ranging from 400 to $800 \mathrm{~nm}$. To demonstrate the ability of controlling the number of TMD layers by our dispersion process with amine-terminated polymers, we varied the centrifuge rate. Statistical analysis of various samples with different centrifuge rates shows that the mean number of layers decreases with the rate and at an optimized condition, a few layered TMDs dispersion was obtained (Supplementary Fig. 17). As expected, bulk and many layers of $\mathrm{MoSe}_{2}$ nanosheets prepared by low centrifugation rate do not exhibit any noticeable PL. In addition, electron microscopy results of both bulk and exfoliated TMDs including $\mathrm{MoS}_{2}, \mathrm{WS}_{2}, \mathrm{WSe}_{2}, \mathrm{ReS}_{2}, \mathrm{ZrTe}_{2}$ and $\mathrm{NbSe}_{2}$ with $\mathrm{PS}-\mathrm{NH}_{2}$ in toluene evidence the universality of our method (Supplementary Fig. 18).

NIR photodetection of flexible $\mathrm{MoSe}_{2}$ nanosheets with PS-NH . To prepare a flexible composite film for use in high performance photodetectors, a suspension of $\mathrm{MoSe}_{2}$ nanosheets modified with $\mathrm{PS}-\mathrm{NH}_{2}$ was carefully poured on a filter paper through which the solvent with non-interacted $\mathrm{PS}-\mathrm{NH}_{2}$ chains was quickly removed. A micron-thick composite film was developed on the filter paper, as shown in Fig. 3a. The surface and crosssectional structures of the composite film exhibit that few-layer $\mathrm{MoSe}_{2}$ nanosheets were stacked with each other, in which the normal surface of the nanosheets is preferentially aligned parallel to the film normal direction. Subsequent thermal deposition of metal electrodes gave rise to mechanically flexible arrays of twoterminal, parallel-type photodetectors, as schematically depicted in Fig. 3a. On illumination with a NIR laser with a wavelength of 1,064 nm, our composite film became conductive due to photoexcited carriers in the exfoliated $\mathrm{MoSe}_{2}$ nanosheets. The photocurrent increases with increasing laser power, yielding a maximum $I_{\text {on }} / I_{\text {off }}$ ratio of $\sim 10^{5}$ at a power density and bias 
a
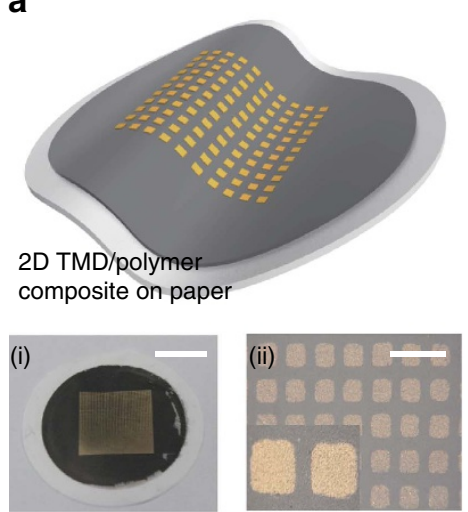
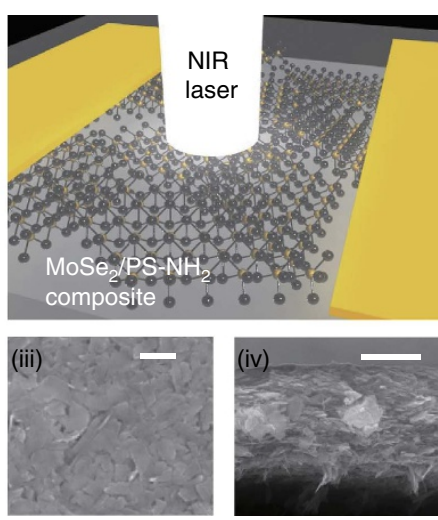
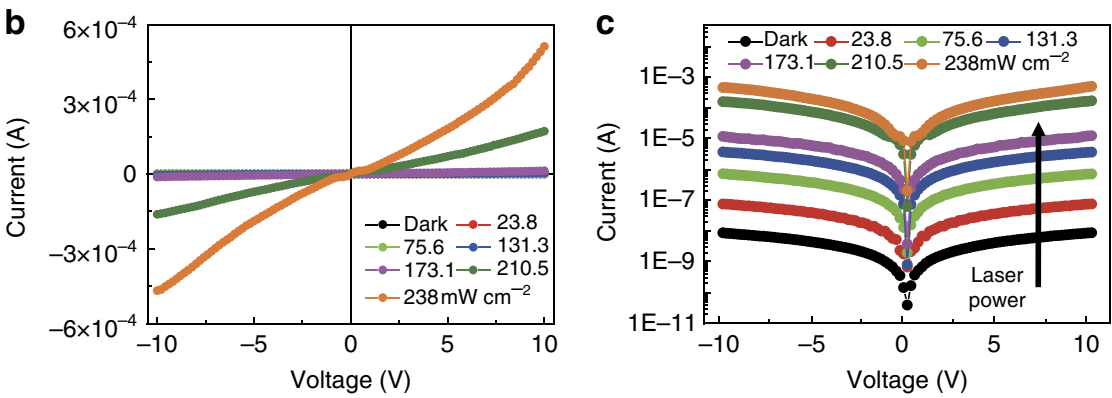

d
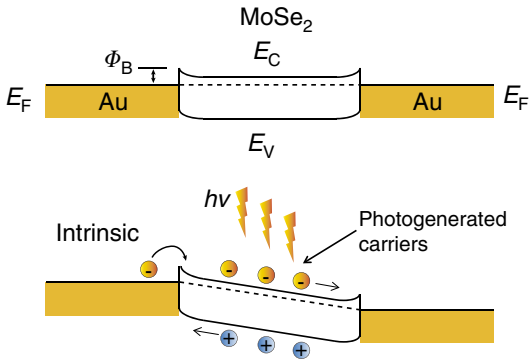

e

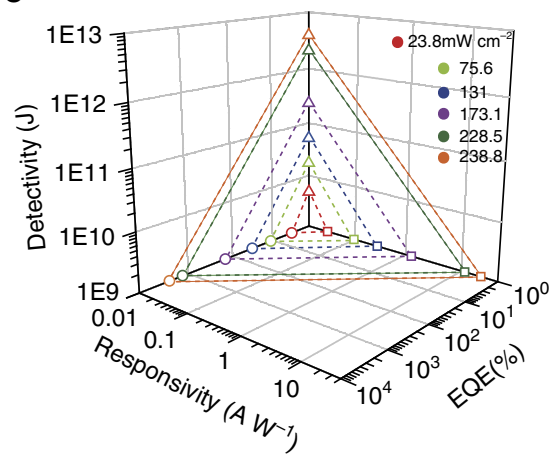

$\mathbf{f}$

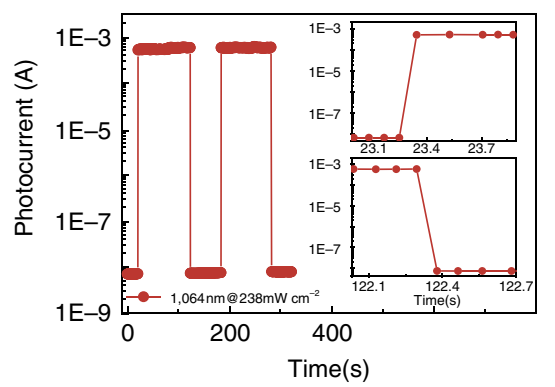

g

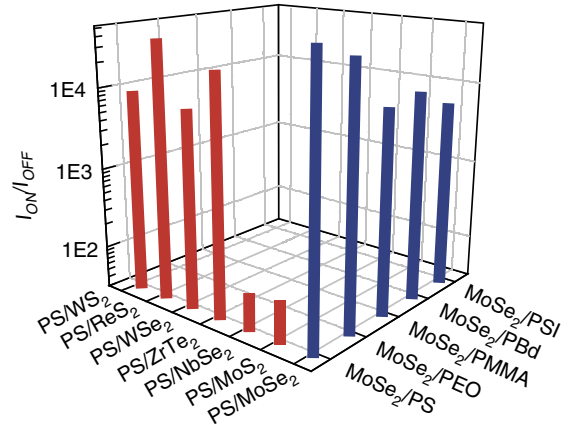

Figure 3 | NIR photodetection performance of thin TMD composite films. (a) Schematic illustration and photographs of the two-terminal, parallel-type photodetector device cells consisting of $\mathrm{MoSe}_{2}$ nanosheets exfoliated with $\mathrm{PS}-\mathrm{NH}_{2}$. The composite film was deposited on nylon membrane filter paper by a vacuum filtration method. SEM images of the surface and cross-sectional structure of the composite film reveal that few-layer MoSe $\mathrm{e}_{2}$ nanosheets are stacked with each other with the normal surface of the nanosheets preferentially aligned parallel to the film normal direction. (Scale bars: (i) $6 \mathrm{~mm}$; (ii) $500 \mu \mathrm{m}$ and (iii and iv) $500 \mathrm{~nm}$ ) (b) Linear and (c) semi-log scale $I-V$ characteristics of the $\mathrm{MoSe}_{2}$ photodetector in the dark and under different light intensities of NIR light at a wavelength of $1,064 \mathrm{~nm}$ with a bias voltage of $\pm 10 \mathrm{~V}$. The photocurrent increases with increasing light intensity due to photoexcited carriers in the exfoliated $\mathrm{MoSe}_{2}$ nanosheets. (d) Band diagram of the $\mathrm{MoSe}_{2}$ photodetector device. (e) The corresponding responsivity, specific detectivity and external quantum efficiency values of the photodetector as a function of the NIR intensity at a bias voltage of $9 \mathrm{~V}$. (f) Photoswitching behaviour of the photodetector under alternating ON and OFF NIR light with an intensity of $238 \mathrm{~mW} \mathrm{~cm}^{-2}$. Both the switch-ON and -OFF times of the detector were $\sim 100 \mathrm{~ms}$. (g) Ratios of the photocurrent to the dark current of the $\mathrm{MoSe}_{2}$ composites with different amine-terminated polymers. The current ratios of various TMD composites with $\mathrm{PS}-\mathrm{NH}_{2}$ are also shown. All of the values were obtained at a light intensity of $238 \mathrm{~mW}^{-2}$. 
voltage of $238 \mathrm{~mW} \mathrm{~cm}^{-2}$ and $10 \mathrm{~V}$, respectively, as shown in Fig. 3b,c (Supplementary Fig. 19).

There exist mainly three sources of photocurrent arising from TMDs on visible and NIR exposure: (1) photoconduction by photo-induced band excited carriers that can be dissociated into free electrons and holes thermally or by a large electric field; and (2) one by photo-excited carriers decaying into heat that makes TMDs warm, resulting in the reduction of electrical resistance, that is, bolometric photocurrent. (3) Photothermoelectric generation of current by light illumination across metal-TMD interface. In our TMD composite film, we believe that the model by the photo-induced band excitation was dominant while both bolometric and photothermoelectric effect are very marginal (Supplementary Fig. 20). As schematically shown in a band diagram of our two-terminal device of Fig. 3d, the photo-excited carriers in conduction band of $\mathrm{MoSe}_{2}$ are drifted to Au electrode with quasi-Ohmic contact under bias field ${ }^{4,18,46,47}$.

The amine-terminated polymer matrix may further promote exciton dissociation on NIR exposure by two possible mechanisms as evidenced in polymer composites with networked semiconducting carbon nanotubes ${ }^{48}$ : (1) Exciton separation by thermal energy built up in the insulating polymer around TMDs; and (2) dissociation by enhanced local electric field at the interface of the insulating polymer and TMDs due to the potential barrier formed by the insulating polymer (Supplementary Note 3). The photodetection performance is independent of film thickness ranging from $500 \mathrm{~nm}$ to $2 \mu \mathrm{m}$, which implies that the laser was able to penetrate through the entire micron-thick film (Supplementary Fig. 21). In addition, no degradation of PS- $\mathrm{NH}_{2}$ was observed on NIR and visible laser exposure (Supplementary Fig. 22).

The photocurrent $\left(I_{\mathrm{ph}}\right)$ arising from photo-excited photocarriers has in general the relation of $I_{\mathrm{ph}} \propto P^{\alpha}$, and in consequence the current linearly increases with laser power in both log scale ${ }^{13}$. In our system, a similar linear relation was observed in the low power regime but the linearity was deviated at high power $>200 \mathrm{~mW} \mathrm{~cm}^{-2}$ with another slope (Supplementary Fig. 19). The rapid increase of photocurrent at high power illumination is known as superlinearity dependence, which has been found in several materials systems but no satisfactory understanding has been made ${ }^{49-53}$. Recently, the superlinear photoresponse was also observed in TMD system in which CVD-grown monolayers of chemically alloyed TMDs exhibited very strong superlinearity in particular at high power regimes, which is similar to our results ${ }^{54}$. The superlinear behaviour at high light intensity was explained using a simplified model with three different types of recombination centres in which initially empty and filled intragap states exist close to the conduction and valence band, respectively. At increased laser intensity, the occupancy change of these centres arising from shifts in the quasi Fermi levels makes the carrier lifetime longer and in turn the recombination rate slower, leading to superlinear photocurrent.

Both the specific detectivity $\left(D^{*}\right)$ and external quantum efficiency values ${ }^{55}$ of our detector increased with increasing laser power up to $4 \times 10^{12}$ Jones and $3 \times 10^{3}$, respectively, as shown in Fig. 3e (Supplementary Note 3). The maximum photoresponsivity of our device was $\sim 16 \mathrm{~A} \mathrm{~W}^{-1}$ at a power intensity of $238 \mathrm{~mW} \mathrm{~cm}^{-2}$. Notably, our NIR photodetector arrays demonstrated very high cell-to-cell and batch-to-batch reliabilities (Supplementary Fig. 23). The device also exhibited a very fast current switching feature in which a sharp response and decay of photocurrent within $\sim 100$ ms was observed on turn-ON and -OFF of the pulsed laser illumination, as shown in Fig. $3 \mathrm{f}$ (Supplementary Fig. 24). In conventional TMD phototransistors (air-exposed TMDs as a channel material), the photo-switching characteristics are primarily determined by the photoconductive effect $^{56}$. In this case, the ON/OFF photocurrent ratio is strongly deteriorated with increasing switching cycle. This is because the air-exposed TMD channel exhibits strong charge-transfer effect between TMDs and the adsorbed $\mathrm{H}_{2} \mathrm{O}$ or $\mathrm{O}_{2}$, such that more photo-induced carriers contribute to the charge transfer with decreasing the switching cycle. On the contrary, our photoresponse shows nearly identical ON/OFF photocurrent ratio regardless of the switching cycle (Supplementary Fig. 24). The near zero variation of ON/OFF photocurrent ratio with switching cycle is possibly because the TMD nanosheets are protected by thin polymer composites dispersed in the matrix, that is, TMDs are not exposed to the air. Dark current measured in air was not different from that under vacuum (Supplementary Fig. 24). Similar to the encapsulated TMD devices ${ }^{57}$, the composite amine-terminated polymers effectively prevent from adsorbing $\mathrm{H}_{2} \mathrm{O}$ or $\mathrm{O}_{2}$ redox couple, suppressing the charge-transfer effect, with which we are able to achieve stable photodetection operation.

The performance of our $\mathrm{MoSe}_{2} / \mathrm{PS}-\mathrm{NH}_{2}$ composite films considering the $I_{\mathrm{on}} / I_{\text {off }}$ ratio, detectivity and response time is even comparable to the results of previous works of a visible photodetector made of single- or few-layer TMDs. Although the switching shorter than $100 \mathrm{~ms}$ were not able to be examined due to the limitation of our switching facility, the switching performance of our composite film is very comparable to that with the monolayer device mechanically cleaved ${ }^{13}$. For the detailed switching dynamics, we provide a zoom-in behaviour in Fig. 3f. Only fast rising transient is observed, implying that the increased photocurrent is largely determined by the photoelectric effect, that is, band-to-band excitation, without the reversible charge transfer from adsorbed molecules to TMD nanosheets. The OFF transient is similar to the ON transient, and comparable to other published results ${ }^{13,16,17,18,46}$, suggesting that a fast band-to-band recombination is dominant with absence of the charge-transfer effect.

NIR photodetectors consisting of $\mathrm{MoSe}_{2}$ nanosheets were readily fabricated with other amine-terminated polymers such as PEO, poly(methyl methacrylate), PB and PIS, as shown in Fig. $3 g$ (Supplementary Fig. 25). All of the $\mathrm{MoSe}_{2}$ composites showed excellent photodetection with a high $I_{\text {on }} / I_{\text {off }}$ ratio $\left(10^{4}\right)$ and a detectivity $>10^{12}$, similar to $\mathrm{PS}-\mathrm{NH}_{2}$. It is also straightforward to develop PS- $\mathrm{NH}_{2}$-based composite films containing various TMDs including $\mathrm{MoS}_{2}, \mathrm{WS}_{2}, \mathrm{ReS}_{2}, \mathrm{WSe} \mathrm{ZrTe}_{2}$ and $\mathrm{NbSe}_{2}$. All of the photodetectors responded on NIR illumination with little variation of the detection performance, which depends on the characteristic photoelectronic properties of the TMDs (Supplementary Fig. 26).

Band-selective photodetection of mixed TMDs with PS- $\mathrm{NH}_{2}$. More interestingly, our solution process is capable of fabricating composite films with various TMDs in the same polymer matrix, that is, PS- $\mathrm{NH}_{2}$, and this method offers a convenient route for band-selective photodetection by simple mixing of two or more different TMDs (Supplementary Note 4). For instance, the excellent photodetection properties of $\mathrm{MoSe}_{2}$ and $\mathrm{MoS}_{2}$ on illumination at $1,064 \mathrm{~nm}$ and $532 \mathrm{~nm}$, respectively, immediately suggest that the performance of a photodetector can be tuned by mixing two TMDs in a PS- $\mathrm{NH}_{2}$ matrix (Supplementary Fig. 27). Moreover, our approach allows for a novel photodetector that can detect over a broad optical spectrum range in both visible and NIR regions, as schematically shown in Fig. 4a. Solution-blended composite films of $\mathrm{MoSe}_{2}$ and $\mathrm{MoS}_{2}$ in a PS- $\mathrm{NH}_{2}$ matrix with different compositions showed homogeneous mixing of the two TMDs, as confirmed by energy dispersive X-ray spectroscopy (EDX) in Fig. 4b (Supplementary Figs 28-30). 

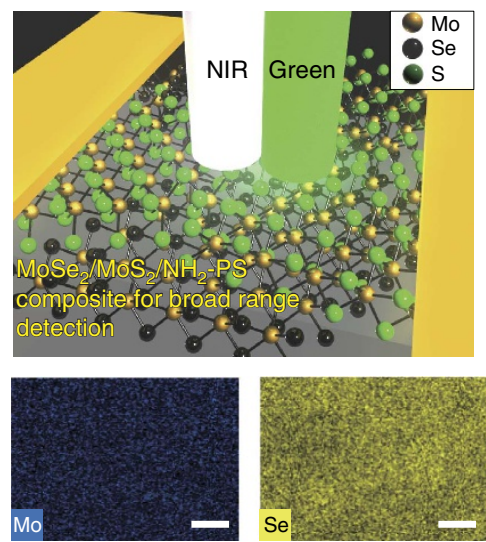

C
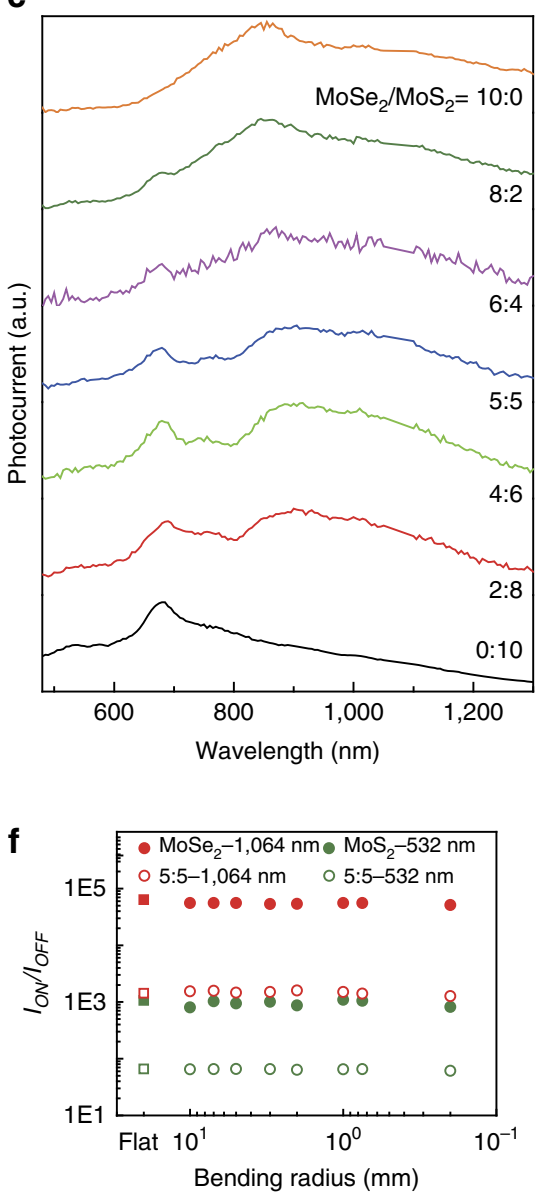

b
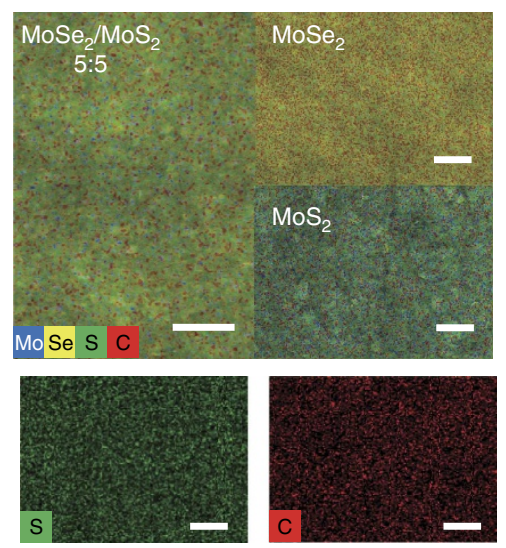

d

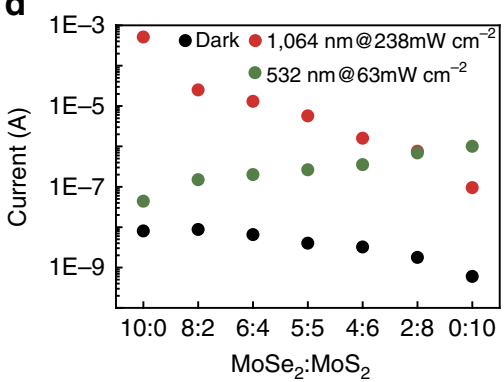

e

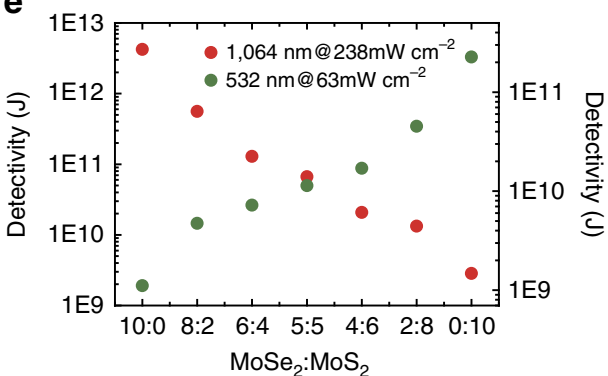

9

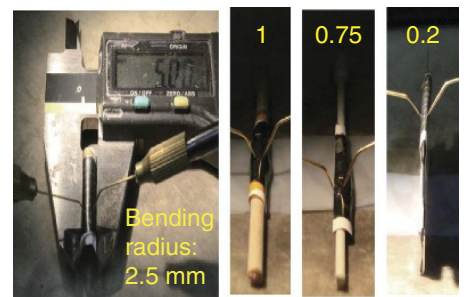

Figure 4 | Flexible TMD composite films for band-selective photodetection. (a) Schematic illustration of the photodetector containing a mixture of $\mathrm{MoSe}_{2}$ and $\mathrm{MoS}_{2}$ nanosheets exfoliated with PS-NH . (b) Energy dispersive X-ray spectroscopy (EDX) mapping of blended $\mathrm{MoS}_{2} / \mathrm{MoSe}_{2}(5: 5), \mathrm{MoSe}_{2}$ and $\mathrm{MoS}_{2}$ films with PS-NH . Elemental mapping of the individual atoms in the $\mathrm{MoS}_{2} / \mathrm{MoSe}_{2}$ (5:5) film are also showed. (Mo, Blue; Se, Yellow; S, Green; C, Red). The Se and S atoms are uniformly distributed over the composite film, which implies that both $\mathrm{MoSe}_{2}$ and $\mathrm{MoS}_{2}$ are mixed homogeneously. (In all cases: the scale bar, $2 \mu \mathrm{m}$ ) (c) Photoresponse performance of blended composites with different mixing ratio of $\mathrm{MoSe}_{2}$ and $\mathrm{MoS}_{2}$ with PS-NH $\mathrm{H}_{2}$ as a function of wavelength in the visible to NIR. (d) Photocurrent and (e) specific detectivity as functions of the composition of the MoSe $e_{2}$ and MoS $\mathrm{S}_{2}$ mixtures embedded in PS- $\mathrm{NH}_{2}$ at wavelengths of $532 \mathrm{~nm}$ and $1,064 \mathrm{~nm}$ with light intensities of $63 \mathrm{~mW} \mathrm{~cm}^{-2}$ and $230 \mathrm{~mW} \mathrm{~cm}^{-2}$, respectively. (f) Ratios of the photocurrent to the dark current of the detectors containing $\mathrm{MoSe}_{2}, \mathrm{MoS}_{2}$ and blended $\mathrm{MoSe}_{2} / \mathrm{MoS}_{2}$ (5:5) composites with PS-NH $\mathrm{N}_{2}$ as a function of the bending radius. $\mathrm{MoSe}_{2}$ and $\mathrm{MoS}_{2}$ composites were examined at a wavelength of $1,064 \mathrm{~nm}$ with a light intensity of $230 \mathrm{~mW} \mathrm{~cm}^{-2}$ and $532 \mathrm{~nm}$ with a light intensity of $63 \mathrm{~mW} \mathrm{~cm}^{-2}$, respectively. On the other hand, blended $\mathrm{MoSe}_{2} / \mathrm{MoS}_{2}$ (5:5) composite film was investigated at both the wavelengths of $1,064 \mathrm{~nm}$ and $532 \mathrm{~nm}$ with a light intensity of $230 \mathrm{~mW} \mathrm{~cm}^{-2}$ and $63 \mathrm{~mW} \mathrm{~cm}^{-2}$, respectively. (g) Photographs of in situ measurements of the flexible photodetectors at different bending radii. 
To demonstrate broadband photoresponse of our blended composites more in detail, we employed a tunable and highly selective laser system (Supplementary Fig. 31) and examined photoresponse performance of blended composites with different mixing ratio of $\mathrm{MoS}_{2}$ and $\mathrm{MoSe}_{2}$ as a function of wavelength as shown in Fig. 4c. The results clearly show that the wavelength at the maximum photocurrent was controlled by the blend ratio. The band-selective photodetection performance of solutionblended composite films of $\mathrm{MoSe}_{2}$ and $\mathrm{MoS}_{2}$ in a PS- $\mathrm{NH}_{2}$ matrix with different compositions was examined at two representative wavelengths-1,064 and $532 \mathrm{~nm}$. The photocurrent and photodetectivity arising from NIR almost linearly decrease while those from visible light increase with increasing amount of $\mathrm{MoS}_{2}$ in the composites, as shown in Fig. 4d,e, respectively (Supplementary Fig. 32). The results clearly indicate that broadband detection is possible, for example, by using a $\mathrm{MoS}_{2} / \mathrm{MoSe}_{2}$ (5:5) composite film. Blended composite films (Supplementary Fig. 33) instantly respond to both 1,064 and $532 \mathrm{~nm}$ light at the millisecond level, which is very similar to a $\mathrm{MoSe}_{2}$ composite (Fig. 3f).

Our TMD composite photodetector demonstrated high mechanical flexibility. The arrays of photodetectors fabricated on conventional filter paper are readily bendable and photocurrent was detected in situ under various bending conditions, as shown in Fig. 4g. Figure $4 \mathrm{f}$ shows three representative composite films with PS- $\mathrm{NH}_{2}$ : neat $\mathrm{MoSe}_{2}, \mathrm{MoS}_{2}$ and blended $\mathrm{MoS}_{2} / \mathrm{MoSe}_{2}$ (5:5). For all three composites, the initial $I_{\text {on }} / I_{\text {off }}$ ratio values barely changed as a function of the bending radius. The values were still maintained even at a bending radius of $\sim 200 \mu \mathrm{m}$. Our mechanically flexible TMD composite photodetectors are also resistant to multiple and repeated deformation. After 1,000 bending cycles at a bending radius of $1 \mathrm{~mm}$, the devices worked properly without any significant deterioration of performance (Supplementary Fig. 34).
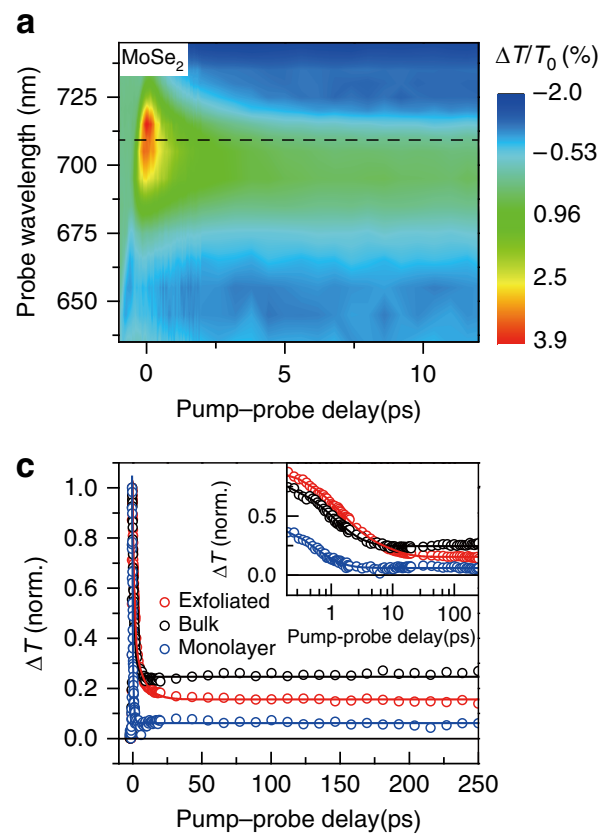

\section{Discussion}

The origin of photocurrent from $\mathrm{MoS}_{2}$ and $\mathrm{MoSe}_{2}$ nanosheets in composites was further revealed by the behaviour of the photoinduced, carrier-relaxation dynamics of the films. Being strongly quantum confined nature of TMD nanosheets, a well-established fact is that TMDs become a direct-gap semiconductor when the thickness is reduced down to a monolayer limit, and the band-toband exciton generation/recombination is dominant. At the same time, the increased surface-to-volume ratio leads to fact that the photo-induced exciton recombination suffers enhanced nonradiative recombination, where such changes are strongly dependent of the number of layers. Thus, understanding the photo-induced response of TMD nanosheets and the photodetector performance requires detailed information on the lightinduced, time-dependent exciton dynamics. It is known that for 2D TMDs, the non-radiative recombination contains multiple exponential decay components, typically in the sub-nanosecond time scale, which are not easily accessible by conventional electronic measurements. In addition, ultrafast spectroscopy measurements can provide not only qualitative information on the layer-number-dependent non-radiative recombination, but also afford quantitative evaluation on the extracted lifetime. Two sets of ultrafast time-resolved pump-probe spectroscopy measurements were performed: optical-pump and optical-probe (OP-OP) and optical-pump and THz-probe (OP-TP) spectroscopy (Supplementary Fig. 35).

To examine the effectiveness of liquid exfoliation of individual TMDs and the corresponding photoresponse of uniformly blended TMDs composite films, we first performed the spectrally and temporally resolved OP-OP measurement on the individual $\mathrm{MoS}_{2}$ and $\mathrm{MoSe}_{2}$ nanosheets modified with PS- $\mathrm{NH}_{2}$, as shown in Fig. 5a,b, and the results are later compared with those from the blended $\mathrm{MoS}_{2} / \mathrm{MoSe}_{2}$ composite (Supplementary Note 5). Here ultrashort $400-\mathrm{nm}$ pump pulse excited the carriers into the
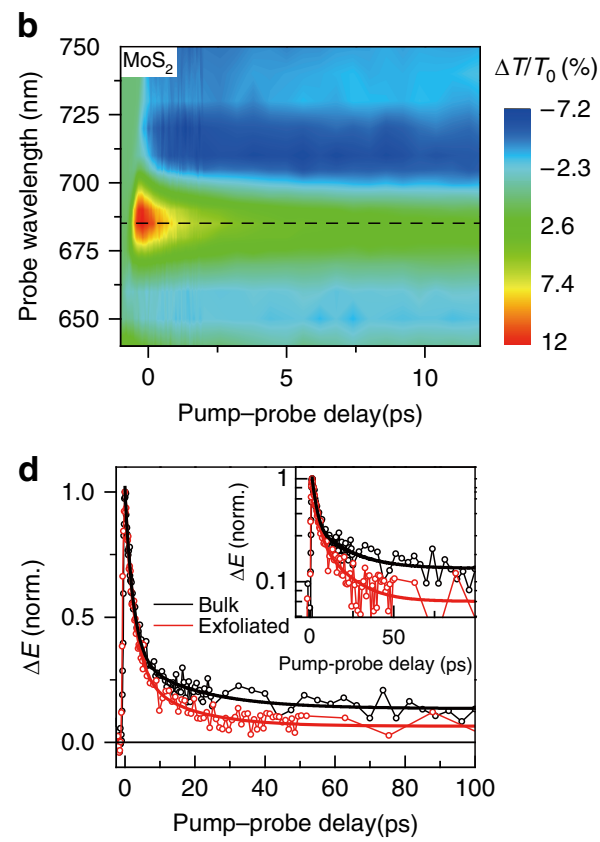

Figure 5 | Ultrafast carrier-relaxation dynamics of the TMD composite films. Two-dimensional plots of transient differential transmission $\Delta T / T_{0}$ spectra of the TMD/polymer film. Each plot represents the measurement result-(a) $\mathrm{MoSe}_{2}$ and (b) $\mathrm{MoS}_{2}$. (c) Time-resolved optical-pump and optical-probe data. Normalized $\Delta T$ dynamics are shown for polymer-exfoliated $M_{o} S_{2}$ nanosheets (red), bulk (black) and monolayer MoS 2 (blue). Inset: $\Delta T$ dynamics plotted with a log scale on $x$-axis. (d) Transient dynamics measured by the optical-pump and THz-probe spectroscopy. Pump-induced field change ( $\Delta E$ ) of the bulk and polymer-exfoliated $\mathrm{MoS}_{2}$ are plotted as a function of the pump-probe delay. Both of the signals were fitted with a bi-exponential decay. Inset: transient $\mathrm{THz}$ dynamics plotted on the log scale to emphasize the slow decay dynamics of the exfoliated sample. 
continuum, and the broadband white-light pulse was used to probe the spectrally resolved exciton transients. For $\mathrm{MoSe}_{2}$ nanosheets, Fig. 5a shows pronounced peak at $710 \mathrm{~nm}$ (dashed line), that is, $A$-exciton resonance of $\mathrm{MoSe}_{2}$. After a few picosecond decays $(\sim 5 \mathrm{ps})$, negative signal $\left(\Delta T / T_{0}<0\right)$ was observed below/above the $A$-exciton resonance. The layernumber-dependent dynamics show clear difference on the carrier-relaxation dynamics between the exfoliated, bulk and monolayer $\mathrm{MoSe}_{2}$ as shown in Fig. 5c (Supplementary Fig. 36). Being strongly quantum confined $2 \mathrm{D}$ nature of TMD, the large surface-to-volume ratio implies multiple and yet very fast exciton dissociation pathways. On the contrary, the carrier relaxation in bulk typically exhibits much slower responses. Figure $5 \mathrm{c}$ shows that our TMD/polymer composite film shows very different carrier-relaxation dynamics compared with the bulk. This corroborates that our TMD nanosheets are well-exfoliated. Similar spectral feature (negative $\Delta T / T_{0}$ around the $A$ exciton of $\mathrm{MoS}_{2}$ after 5-ps delay) was observed for $\mathrm{MoS}_{2}$ nanosheets, as shown in Fig. $5 b$.

It should be, however, noted that one should be cautious to interpret the optical transient responses since there exist multiple reasons for the observed responses such as exciton linewidth broadening, resonance shift and biexcition formation, together with defect- and Auger-induced recombination. We observed both linewidth broadening and exciton energy shift in our nanocomposite samples but it is not trivial to exactly identify the physical origin of the transient responses from our TMD nanocomposites. For instance, due to the dispersive characters of the $\mathrm{MoS}_{2}$ sheet solution exfoliated with $\mathrm{PS}-\mathrm{NH}_{2}$, inhomogeneous broadening of $\mathrm{A}$ and $\mathrm{B}$ resonances might occur, giving rise to the energy shift. In addition, the 'spectral wing' of B exciton overlapped with that of A exciton for the pump-excited nonequilibrium response may result in the energy shift. Under high pump excitation condition, Auger effect may influence our measured signals. In spite of some unambiguity for interpreting the transient responses, the results sufficiently support our claims that our blended $\mathrm{MoS}_{2} / \mathrm{MoSe}_{2}$ composite is well-dispersed individually, rather than just simply mixing two dissimilar TMD powders, corroborating the band-selective photodetection mechanism of Fig. 4.

The $\mathrm{THz}$ probe provides a straightforward measure of the photo-induced conductivity dynamics due to the low-energy nature of $\mathrm{THz}$ radiation $(1 \mathrm{THz}=4.136 \mathrm{meV}$; Supplementary Note 6). In addition, the OP-TP measurements allowed us to examine the validity of liquid polymer exfoliation of TMDs from the bulk, in which the photogenerated $\mathrm{THz}$ intraband carrier relaxation is expected to show different conductivity dynamics between the exfoliated TMD-polymer nanosheets and the bulk (Supplementary Figs 37 and 38). In Fig. 5d, we directly compare the $\mathrm{THz}$ dynamics of bulk $\mathrm{MoS}_{2}$ (black) with the few-layer $\mathrm{MoS}_{2}$ nanosheets exfoliated with $\mathrm{PS}-\mathrm{NH}_{2}$ (red). Immediately after photoexcitation, both the bulk and few-layer $\mathrm{MoS}_{2}$ show almost the same rising dynamics. This rapidly rising signal reflects the extremely fast (within a 1-ps $\mathrm{THz}$ pulse width) intraband carrier relaxation from the continuum to the bound exciton state. For the long decay component, however, the time scale of the two samples is largely different. The inset in Fig. 5d clearly shows that the $\mathrm{MoS}_{2}$-polymer nanosheets possess much faster decay dynamics than that of bulk $\mathrm{MoS}_{2}$, presumably due to the interfacial traps arising from the high surface-to-volume ratio of the few-layer $\mathrm{MoS}_{2}$ nanosheets ${ }^{56,58,59}$. It is worth mentioning that the existing $\mathrm{THz}$ study ${ }^{58}$ performed similar ultrafast $\mathrm{THz}$ experiments and reported the transient decrease of $\mathrm{THz}$ conductivity after optical excitation, which is quite different compared with our measurements. There, the negative conductivity dynamics was attributed to the trion formation in highly doped $\mathrm{MoS}_{2}$ monolayer. Because the trion can be observed in the high doping case, the possibility of trion formation is low for the weakly doped system. In fact, the mechanically exfoliated or CVD-grown $\mathrm{MoS}_{2}$ without any electrostatic doping or chemical doping shows a non-degenerated n-type behaviour, so that it is unlikely that the trion formation is contributed to the $\mathrm{THz}$ conductivity dynamics for our case. The results of both the OP-OP and OP-TP spectroscopy analyses suggest not only the effectiveness of our exfoliation process with amine-terminated polymers but also the independent response of $\mathrm{MoSe}_{2}$ and $\mathrm{MoS}_{2}$ nanosheets in a blended film, which validates our approach for a band-selective detector.

In summary, we demonstrated a scalable platform suitable for fabricating various flexible TMD/polymer composite films in which few-layer TMD nanosheets were properly separated from each other and embedded in a polymer matrix. The pivotal process for the successful platform is the extremely efficient liquid exfoliation of TMDs with primary amine-terminated polymers whose end-functional amines were firmly anchored on the surface of TMDs. More interestingly, our $\mathrm{MoSe}_{2}$ and $\mathrm{MoS}_{2}$ composites were highly photoconductive even at extreme bending radii as low as $200 \mu \mathrm{m}$ on illumination of NIR and visible light, respectively. Furthermore, simple solution mixing of $\mathrm{MoSe}_{2}$ and $\mathrm{MoS}_{2}$ gave rise to blended composite films in which the photodetection properties were tunable. The $\mathrm{MoS}_{2} / \mathrm{MoSe}_{2}$ (5:5) film showed broad range photodetection suitable for both visible and NIR spectra, as confirmed by photo-induced, carrierrelaxation dynamics.

\begin{abstract}
Methods
Exfoliation of $\mathrm{MoSe}_{2}$ with amine-end-functionalized polymers. TMD powders were purchased from Alfa Aeser and Sigma Aldrich. The amine-terminated polymers and polystyrene homopolymer were procured from Polymer Source Inc., Doval, Canada. The physical properties of the TMD powders and polymers are listed in the Supplementary Tables 1 and 2, respectively. All of the solvents were purchased from Sigma Aldrich. All of the materials were used as received unless otherwise stated. In a typical procedure, $250 \mathrm{mg}$ of bulk $\mathrm{MoSe}_{2}$ powder and $25 \mathrm{mg}$ of PS- $\mathrm{NH}_{2}$ were added into a 30-ml glass vial containing $25 \mathrm{ml}$ of toluene. The solution was sonicated for $45 \mathrm{~min}$ by using a tip sonicator with a 10 -s On pulse and a 5-s OFF pulse at an amplitude of $50 \%$ in an ice bath. The dispersions were allowed to settle for $24 \mathrm{~h}$, and then the top dispersion was decanted and centrifuged for $30 \mathrm{~min}$ at 1,500 r.p.m. to remove the unexfoliated and large particles. After centrifugation, the top half of the dispersion was collected and the concentration of $\mathrm{MoSe}_{2}$ nanosheets was determined by standard gravimetric analysis.
\end{abstract}

Photodetector fabrication. To fabricate the devices, stock solutions were centrifuged for $90 \mathrm{~min}$ at 15,000 r.p.m. to remove the excess non-interacted polymers in the dispersions. Then, the precipitate was collected and dried to remove the solvents. The resultant powder consisted of $\sim 8: 2(\mathrm{w} / \mathrm{w})$ ratio of $\mathrm{MoSe}_{2}$ and $\mathrm{PS}-\mathrm{NH}_{2}$. The desired amount of powder was dispersed in the solvent by bath sonication for $20 \mathrm{~min}$ and then filtered under vacuum onto a nylon membrane filter paper with a pore size of $200 \mathrm{~nm}$ and a diameter of $25 \mathrm{~mm}$. For the fabrication of the mixture of the TMD composite films, first, powder of the individual TMD nanosheets with polymers was dispersed in the solvent. Then, the solutions were mixed together at different weight ratios by a simple solution blending method and deposited on filter paper. The resulting film was dried in a vacuum oven at $60^{\circ} \mathrm{C}$ for $4 \mathrm{~h}$. Parallel Au electrodes with thicknesses of $50 \mathrm{~nm}$ were deposited onto a $\mathrm{MoSe}_{2}$ film by thermal evaporation under a vacuum of $10^{-6}$ torr using a patterned shadow mask. The length and width of the channels were $50 \mu \mathrm{m}$ and $200 \mu \mathrm{m}$, respectively.

Equipment and characterization. A horn probe tip sonicator (VibraCell CVX; $750 \mathrm{~W}$ ) was used to exfoliate the TMDs with amine-terminated polymers. Centrifugation was carried out using a Hettich Mikro 22R centrifuge. The optical absorbance spectra were measured by a ultraviolet-visible-NIR spectrophotometer (JASCO V-530) using 1-cm quartz cuvettes. FT-IR studies were performed using a JASCO FT-IR 300E apparatus (Tokyo, Japan) with $\mathrm{KBr}$ as a standard. The thermogravimetric analysis (TGA) of the samples was carried out using a TA Q500 thermal analyser at a heating rate of $10^{\circ} \mathrm{C} \mathrm{min}{ }^{-1}$ under a nitrogen atmosphere. $\mathrm{X}$-ray photoelectron spectroscopy (K-alpha Thermo VG, .K.) measurements were acquired using a monochromated $\mathrm{Al} \mathrm{X}$-ray source ( $\mathrm{Al} \mathrm{K} \alpha$ line: $1486.6 \mathrm{eV}$ ). Raman and PL measurements (LabRamAramis) were carried out using a 532-nm laser at a power of $0.5 \mathrm{~mW}$ and an exposition time of $10 \mathrm{~s}$. X-ray diffraction patterns were 
recorded using a Dmax/2500-H (Rigaku, Japan). The nanostructures of the TMDs were examined by tapping mode atomic force microscopy (Nanoscope IV Digital Instruments) in the height and phase contrast mode, field emission scanning electron microscopy (FESEM, JEOL JSM-7001F) with an acceleration voltage of $10 \mathrm{kV}$ and high resolution transmission electron microscopy (HRTEM, JEOL $2100 \mathrm{~F}$ ) at $200 \mathrm{kV}$ in the bright field. TEM samples were prepared by drop casting the dispersions on a holy carbon grid, followed by drying under vacuum for $24 \mathrm{~h}$ at $50^{\circ} \mathrm{C}$. Selected area electron diffraction patterns were obtained in the HRTEM analysis. Energy dispersive X-ray (EDX) spectra of the TMD/polymer composite films were obtained using an EDX spectrometer by FESEM with an acceleration voltage of $10 \mathrm{kV}$. The electrical properties of all devices were measured at room temperature in air with a Hewlett-Packard 4145B semiconductor parameter analyser. The $532 \mathrm{~nm}$ and 1,064 $\mathrm{nm}$ wavelength light were generated from different continuous-wave semiconductor diode laser sources, Shanghai dream Lasers Technology Co., Ltd., Model number: SDL-532-005T and Qbic Laser system, Model number: QBFDL-1064-150-1000, respectively. A laser power meter was used to measure the incident power of the laser pulses. The exciton formation and recombination kinetics of the $\mathrm{MoSe}_{2}$ and $\mathrm{MoS}_{2}$ composite film with PS-NH $\mathrm{NH}_{2}$ was measured with ultrafast optical-pump and optical-probe spectroscopy. The photogenerated intraband carrier-relaxation dynamics of few-layer $\mathrm{MoS}_{2}$ nanosheets exfoliated by PS- $\mathrm{NH}_{2}$ was examined with ultrafast OP-TP spectroscopy. The detailed experimental set-up for the ultrafast spectroscopy measurements are shown in Supplementary Figs 35 and 37.

\section{References}

1. Wang, Q. H., Kalantar-Zadeh, K., Kis, A., Coleman, J. N. \& Strano, M. S. Electronics and optoelectronics of two-dimensional transition metal dichalcogenides. Nat. Nanotechnol. 7, 699-712 (2012).

2. Chhowalla, M. et al. The chemistry of two-dimensional layered transition metal dichalcogenide nanosheets. Nat. Chem. 5, 263-275 (2013).

3. Nicolosi, V., Chhowalla, M., Kanatzidis, M. G., Strano, M. S. \& Coleman, J. N. Liquid exfoliation of layered materials. Science 340, 1420-1424 (2013).

4. Radisavljevic, B., Radenovic, A., Brivio, J., Giacometti, V. \& Kis, A. Single-layer $\mathrm{MoS}_{2}$ transistors. Nat. Nanotechnol. 6, 147-150 (2011).

5. Kim, S. et al. High-mobility and low-power thin-film transistors based on multilayer $\mathrm{MoS}_{2}$ crystals. Nat. Commun. 3, 1011 (2012).

6. Wang, H. et al. Integrated circuits based on bilayer $\mathrm{MoS}_{2}$ transistors. Nano Lett. 12, 4674-4680 (2012).

7. Wang, K. et al. Ultrafast saturable absorption of two-dimensional $\mathrm{MoS}_{2}$ nanosheets. ACS Nano 7, 9260-9267 (2013).

8. Ross, J. S. et al. Electrically tunable excitonic light-emitting diodes based on monolayer $\mathrm{WSe}_{2}$ p-n junctions. Nat. Nanotechnol. 9, 268-272 (2014).

9. Pospischil, A. et al. Solar-energy conversion and light emission in an atomic monolayer p-n diode. Nat. Nanotechnol. 9, 257-261 (2014).

10. Stephenson, T. et al. Lithium ion battery applications of molybdenum disulfide $\left(\mathrm{MoS}_{2}\right)$ nanocomposites. Energy Environ. Sci. 7, 209-231 (2014).

11. Voiry, D. et al. Enhanced catalytic activity in strained chemically exfoliated $\mathrm{WS}_{2}$ nanosheets for hydrogen evolution. Nat. Mater. 12, 850-855 (2013).

12. Yin, Z. et al. Single-layer $\mathrm{MoS}_{2}$ phototransistors. ACS Nano 6, 74-80 (2012).

13. Lopez-Sanchez, O., Lembke, D., Kayci, M., Radenovic, A. \& Kis, A. Ultrasensitive photodetectors based on monolayer $\mathrm{MoS}_{2}$. Nat. Nanotechnol. 8, 497-501 (2013).

14. Zhang, W. et al. High-gain phototransistors based on a CVD $\mathrm{MoS}_{2}$ monolayer. Adv. Mater. 25, 3456-3461 (2013).

15. Perea-López, N. et al. Photosensor device based on few-layered $\mathrm{WS}_{2}$ films. $A d v$. Funct. Mater. 23, 5511-5517 (2013)

16. Chang, Y.-H. et al. Monolayer $\mathrm{MoSe}_{2}$ grown by chemical vapor deposition for fast photodetection. ACS Nano 8, 8582-8590 (2014).

17. Xia, J. et al. CVD synthesis of large-area, highly crystalline $\mathrm{MoSe}_{2}$ atomic layers on diverse substrates and application to photodetectors. Nanoscale 6, 8949-8955 (2014).

18. Cunningham, G. et al. Photoconductivity of solution-processed $\mathrm{MoS}_{2}$ films. J. Mater. Chem. C 1, 6899-6904 (2013).

19. Novoselov, K. S. et al. Two-dimensional atomic crystals. Proc. Natl Acad. Sci. USA 102, 10451-10453 (2005).

20. Bertolazzi, S., Brivio, J. \& Kis, A. Stretching and breaking of ultrathin $\mathrm{MoS}_{2}$. ACS Nano 5, 9703-9709 (2011).

21. Castellanos-Gomez, A. et al. Elastic properties of freely suspended $\mathrm{MoS}_{2}$ nanosheets. Adv. Mater. 24, 772-775 (2012).

22. Joensen, P., Frindt, R. F. \& Morrison, S. R. Single-layer $\mathrm{MoS}_{2}$. Mater. Res. Bull. 21, 457-461 (1986)

23. Benavente, E., Santa Ana, M. A., Mendizabal, F. \& Gonzalez, G. Intercalation chemistry of molybdenum disulfide. Coord. Chem. Rev. 224, 87109 (2002).

24. Golub, A. S., Zubavichus, Y. V., Slovokhotov, Y. L. \& Novikov, Y. N. Single-layer dispersions of transition metal dichalcogenides in the synthesis of intercalation compounds. Russ. Chem. Rev. 72, 123-141 (2003).

25. Zheng, J. et al. High yield exfoliation of two-dimensional chalcogenides using sodium naphthalenide. Nat. Commun. 5, 2995 (2014).
26. Coleman, J. N. et al. Two-dimensional nanosheets produced by liquid exfoliation of layered materials. Science 331, 568-571 (2011).

27. Zhou, K. G., Mao, N. N., Wang, H. X., Peng, Y. \& Zhang, H. L. A mixed-solvent strategy for efficient exfoliation of inorganic graphene analogues. Angew. Chem. Int. Ed. 50, 10839-10842 (2011).

28. Zeng, Z. et al. Single-layer semiconducting nanosheets: high-yield preparation and device fabrication. Angew. Chem. Int. Ed. 50, 11093-11097 (2011).

29. Smith, R. J. et al. Large-scale exfoliation of inorganic layered compounds in aqueous surfactant solutions. Adv. Mater. 23, 3944-3948 (2011).

30. Cunningham, G. et al. Solvent Exfoliation of transition metal dichalcogenides: dispersibility of exfoliated nanosheets varies only weakly between compounds. ACS Nano 6, 3468-3480 (2012).

31. May, P., Khan, U., Hughes, J. M. \& Coleman, J. N. Role of solubility parameters in understanding the steric stabilization of exfoliated two-dimensional nanosheets by adsorbed polymers. J. Phys. Chem. C 116, 11393-11400 (2012).

32. Liu, J. Q. et al. Preparation of $\mathrm{MoS}_{2}$-Polyvinylpyrrolidone nanocomposites for flexible nonvolatile rewritable memory devices with reduced graphene oxide electrodes. Small 8, 3517-3522 (2012).

33. Yoo, D., Kim, M., Jeong, S., Han, J. \& Cheon, J. Chemical synthetic strategy for single-layer transition-metal chalcogenides. J. Am. Chem. Soc. 136, 14670-14673 (2014).

34. Velusamy, D. B. et al. High throughput modification of chemically reduced graphene oxides by a conjugated block copolymer in non-polar medium. J. Mater. Chem. 22, 25183-25189 (2012).

35. Gamble, F. R. et al. Intercalation complexes of Lewis bases and layered sulfides: a large class of new superconductors. Science 174, 493-497 (1971).

36. Li, J. et al. Enhanced-electrocatalytic activity of $\mathrm{Ni}_{1-x} \mathrm{Fe}_{x}$ alloy supported on polyethyleneimine functionalized $\mathrm{MoS}_{2}$ nanosheets for hydrazine oxidation. RSC Adv. 4, 1988-1995 (2014).

37. Sumboja, A. et al. Significant electrochemical stability of manganese dioxide/polyaniline coaxial nanowires by self-terminated double surfactant polymerization for pseudocapacitor electrode. J. Mater. Chem. 22, 23921-23928 (2012).

38. Aradi, B., Hourahine, B. \& Frauenheim, T. DFTB +, a sparse matrix-based implementation of the DFTB method. J. Phys. Chem. A 111, 5678-5684 (2007).

39. Porezag, D., Frauenheim, T., Köhler, T., Seifert, G. \& Kaschner, R. Construction of tight-binding-like potentials on the basis of density-functional theory: application to carbon. Phys. Rev. B 51, 12947 (1995).

40. Seifert, G. \& Porezag, D. Frauenheim, calculations of molecules, clusters, and solids with a simplified LCAO-DFT-LDA scheme. Int. J. Quant. Chem. 58, 185-192 (1996).

41. Wilson, J. A. \& Yoffe, A. D. Transition metal dichalcogenides: discussion and interpretation of observed optical, electrical and structural properties. $A d v$. Phys. 18, 193-335 (1969).

42. Tonndorf, P. et al. Photoluminescence emission and raman response of monolayer $\mathrm{MoS}_{2}, \mathrm{MoSe}_{2}$, and $\mathrm{WSe}_{2}$. Opt. Exp. 21, 4908-4916 (2013).

43. Eda, G. et al. Photoluminescence from chemically exfoliated $\mathrm{MoS}_{2}$. Nano Lett. 11, 5111-5116 (2011).

44. Shaw, J. C. et al. Chemical vapor deposition growth of monolayer $\mathrm{MoSe}_{2}$ nanosheets. Nano Res. 7, 1-7 (2014).

45. Lee, Y.-H. et al. Synthesis and transfer of Single-layer transition metal disulfides on diverse substrates. Nano Lett. 13, 1852-1857 (2011).

46. Cunningham, G. et al. Large variations in both dark- and photoconductivity in nanosheet networks as nanomaterial is varied from $\mathrm{MoS}_{2}$ to $\mathrm{WTe}_{2}$. Nanoscale 7, 198-208 (2015).

47. Lembke, D. \& Kis, A. Breakdown of high-performance monolayer $\mathrm{MoS}_{2}$ transistors. ACS Nano 6, 10070-10075 (2012).

48. Pradhan, B. et al. Carbon nanotube-polymer nanocomposite infrared sensor. Nano Lett. 8, 1142-1146 (2008).

49. Rose, A. Recombination processes in insulators and semiconductors. Phy. Rev. 97, 322-333 (1955).

50. Bakr, N. A. Anomalous photoconductive transport properties of As2Se3 films Egypt. J. Sol. 25, 13-21 (2002).

51. Kolomiets, B. T. \& Lyubin, V. M. Photoelectric phenomena in amorphous chalcogenide semiconductors. Phys. Stat. Sol. 17, 11-46 (1973).

52. Irvin, P. et al. Rewritable nanoscale oxide photodetector. Nat. Photonics 4, 849-852 (2010).

53. Feng, W. et al. Synthesis of two-dimensional b-Ga2O3 nanosheets for high-performance solar blind photodetectors. J. Mater. Chem. C 2, 3254-3259 (2014).

54. Klee, V. et al. Superlinear composition-dependent photocurrent in CVD-grown monolayer $\mathrm{MoS}_{2}(1-\mathrm{x}) \mathrm{Se}_{2} \mathrm{x}$ alloy devices. Nano Lett. 15, 2612-2619 (2015).

55 . Gong, X. et al. High-detectivity polymer photodetectors with spectral response from 300 to $1450 \mathrm{~nm}$. Science 325, 1665-1667 (2009).

56. Furchi, M. M., Polyushkin, D. K., Pospischil, A. \& Mueller, T. Mechanisms of photoconductivity in atomically thin $\mathrm{MoS}_{2}$. Nano Lett. 14, 6165-6170 (2014). 
57. Late, D. J. et al. Hysteresis in single-layer $\mathrm{MoS}_{2}$ field effect transistors. ACS Nano 6, 5635-5641 (2012).

58. Lui, C. et al. Trion induced negative photoconductivity in monolayer $\mathrm{MoS}_{2}$. Phys. Rev. Lett. 113, 166801 (2014).

59. Sun, D. et al. Observation of rapid exciton-exciton annihilation in monolayer molybdenum disulfide. Nano Lett. 14, 5625-5629 (2014).

\section{Acknowledgements}

This work was supported by the third Stage of the Brain Korea 21 Plus Project in 2014 and the National Research Foundation of Korea(NRF) grant funded by the Korea government (MEST) (No.2014R1A2A1A01005046). We gratefully acknowledge the financial support provided by the Agency for Defense Development under the contract UD110050GD. S.C. and H.C. were supported by NRF of Korea through MSIP (NRF-2011-0013255).

\section{Author contributions}

C.P., H.C. and D.B.V. conceived and designed the experiments. D.B.V. performed the dispersion, and fabrication of the photodetector devices. G.S. and S.M.C. performed the morphology characterization. D.B.V., R.H.K., R.K., S.H.K., S.H.C., I.H., J.L. and K.O were involved in the measurements and characterization of the photodetector devices. S.C. carried out the ultrafast spectroscopy measurements. J.H. carried out the molecular dynamics simulation. C.P. and H.C. supervised the project. C.P., H.C., and D.B.V. analysed the data and wrote the paper. All authors discussed the results and commented on the manuscript.

\section{Additional information}

Supplementary Information accompanies this paper at http://www.nature.com/ naturecommunications

Competing financial interests: The authors declare no competing financial interests

Reprints and permission information is available online at http://npg.nature.com/ reprintsandpermissions/

How to cite this article: Velusamy, D. B. et al. Flexible transition meta dichalcogenide nanosheets for band-selective photodetection. Nat. Commun. 6:8063 doi: 10.1038/ncomms9063 (2015)

(c) (i) This work is licensed under a Creative Commons Attribution 4.0 International License. The images or other third party material in this article are included in the article's Creative Commons license, unless indicated otherwise in the credit line; if the material is not included under the Creative Commons license, users will need to obtain permission from the license holder to reproduce the material. To view a copy of this license, visit http://creativecommons.org/licenses/by/4.0/ 